\title{
STUDY OF ENZYME POLYMORPHISM AND HAEMOGLOBIN PATTERNS AMONGST SIXTEEN TRIBAL POPULATIONS OF CENTRAL INDIA (ORISSA, MADHYA PRADESH, AND MAHARASHTRA)
}

\author{
Ketaki DaS, ${ }^{1}$ Monami Roy, ${ }^{1}$ M.K. DAS, ${ }^{1}$ \\ P.N. SAhU, ${ }^{2}$ S.K. BhatTacharya, ${ }^{1}$ K.C. Malhotra, ${ }^{1}$ \\ B.N. MUKHERJEE, ${ }^{1}$ and H. WALTER ${ }^{3}$ \\ ${ }^{1}$ Anthropometry and Human Genetics Unit, Indian Statistical Institute, \\ 203 Barrackpore Trunk Road, Calcutta 700 035, India \\ ${ }^{2}$ Department of Anthropology, Sambalpur University, \\ Burla, Sambalpur, Orissa, India \\ ${ }^{3}$ Department of Human Biology, University of Bremen, \\ Bremen, Germany
}

\begin{abstract}
Summary A survey was conducted to study the genetic differentiation among 16 tribal groups of Orissa, Madhya Pradesh, and Maharashtra belonging to different ethnic and linguistic affiliations. Sixteen hundred and fifteen blood samples from both sexes were tested for 5 red cell enzyme systems: ACP, ESD, PGD, GLO, LDH, and Hb pattern. Three hundred and nineteen male individuals were tested for G-6-PD enzyme deficiency. The distribution of the enzyme markers and $\mathrm{Hb}$ show a range of variation which are more or less within the Indian range. Cases of homozygous HbSS were detected in all the tribes except 3 tribes in Orissa. Two cases of LDH Cal-1 homozygote were found in two Dravidian language speaking Orissa tribes. The $\chi^{2}$-values for testing the homogeneity of gene frequencies indicate a non-significant heterogeneity for all alleles in the individual system. Within population diversity seems to be larger than between population diversity. The degree of over all genetic differentiation as measured by $\mathrm{G}_{\mathrm{ST}}$ value is $0.0154 \pm 0.0071$.
\end{abstract}

Key Words genetic polymorphism, tribal groups, Central India, red cell enzymes, haemoglobin, gene diversity

\section{INTRODUCTION}

The Indian subcontinent comprises about 40,000 endogamous population groups including 427 tribal groups. The tribal groups numbering about 51,628,638

Received February 15, 1993; Revised version accepted May 17, 1993. 
individual constitute $7.76 \%$ of the total population of India (Census of India, 1971). Their population size ranges from very small (Toda, Toto, Andamanese, Onges) to very large (Santal, Bhil, Gond, etc.) groups. In fact, some of the small tribes are likely to become extinct. These tribal groups are quite heterogeneous in respect of their origin, relegion, cultural practices, and linguistic affiliation, and they are found in almost all parts of India having a high concentration in Central India. Among these tribes numerically most dominating are the Gond in Medhya Pradesh, Maharashtra, and Andhra Pradesh; the Bhills of Maharashtra, Madhya Pradesh, Gujrat, and Rajasthan; and the Santal of Bihar, Orissa, and West Bengal. According to morphological and somatoscopic characters the tribes of India can be broadly classified into three ethnic types as Mongoloid, Proto-Australoid, and Europoid. The Mongoloids are mainly found in the northern and north-eastern Himalayas and speak language of the Tibeto-Chinese language family. The ProtoAustraloids are mainly concentrated in the hills and plateaus of Central India and the Indo-Gangetic plains, as well as in some parts of Southern and Northern India. Linguistically, they belong to Austro-Asiatic, Dravidian, and Indo-European language families. The south western Indian tribes speak mostly Dravidian languages (Mukherjee, 1984).

The tribes are in various stages of development with primitive means of livelihood like hunting and food gathering to advanced and modern one. Most of the tribes follow rigid endogamy including various types of consanguinity practices. The tribes show so much diversities in their physical make-up, language, social and cultural practices etc., that it would be interesting to study the ongoing genetic differentiation amongst them. Biochemical genetic markers are very useful in the study of the processes of the genetic differentiation within and among them as well as to the understanding of microevolutionary process in Man. Not much informations on the distribution of genetic markers in the Indian tribes are available, particularly in the Central India belt (Walter et al., 1992). A project was therefore undertaken to study various biochemical polymorphisms in 16 tribal groups of three states namely, Orissa, Madhya Pradesh, and Maharashtra of Central India, belonging to different ethnic socio-cultural and linguistic affiliation with the following aims and objects; (1) to find out the allele distribution of five red cell enzyme markers as well as the haemoglobin types, (2) to analyse the genetic diversity within and between the 16 tribes, and (3) to study the extent of genetic relationship between them. The ethnological descriptions and the geographical location of the 16 tribal groups understudy are given elsewhere (Walter et al., 1992).

\section{MATERIALS AND METHODS}

Villages in several districts of three states (Orissa, Madhya Pradesh, and Maharashtra) were chosen for the study of 16 tribal population, namely, (1) Desia Khonds, (2) Raj Gonds (Orissa), (3) Savaras, (4) Gadabas (Bade), (5) Konda Doras, 
(6) Kuvi Khonds, (7) Parojas (Jhoria), (8) Binjhals, (9) Kisans, (10) Bhatras, (11) Dhurwas, (12) Halbas, (13) Marias (Dandami), (14) Murias, (15) Maria Gonds, and (16) Raj Gonds (Maharashtra). Relevant demographic and linguistic data location etc. of each tribe are given in the Table 1 including the number of individuals studied. Six red cell enzyme systems and haemoglobin types were studied: (1) Acid phosphatase (ACP), (2) esterase-D (ESD), (3) 6-phosphogluconate dehydrogenase (PGD), (4) glyoxalase (GLO), (5) lactate dehydrogenase (LDH), (6) G-6-PD deficiency, and (7) haemoglobin types $(\mathrm{Hb})$. Sixteen hundred and fifteen blood samples were collected from the individuals of both sexes of the 16 tribal groups from finger tips in the field during January to March 1989. Separation of blood cells and serum were done in the field and transported by air in ice box to the Anthropometry and Human Genetics Unit of the Indian Statistical Institute, Calcutta, where the screening for enzyme polymorphism were performed. The red cell isozymes were determined following the techniques of Swallow et al. (1973) for ACP; Fields and Parr (1963) for PGD; Kompf et al. (1975) for GLO-I; Das et al. (1972) for LDH; and

Table 1. The names, geographical location, population size, and linguistic affiliation of the 16 tribes studied from Central India together with number of individuals studied from each tribe.

\begin{tabular}{|c|c|c|c|c|c|c|}
\hline State & District & Population & $\begin{array}{c}\text { No. of } \\
\text { population } \\
\text { in the } \\
\text { district } \\
\text { (Census, 1971) }\end{array}$ & $\begin{array}{l}\text { No. of } \\
\text { individ- } \\
\text { uals } \\
\text { studied }\end{array}$ & $\begin{array}{l}\text { Linguistic } \\
\text { group }\end{array}$ & $\begin{array}{l}\text { Sub- } \\
\text { group }\end{array}$ \\
\hline \multirow[t]{9}{*}{ Orissa } & \multirow[t]{3}{*}{ Kalahandi } & 1. Deshia Khonds & 114,644 & 110 & Dravidian & Kui \\
\hline & & 2. Raj Gonds & 123,778 & 107 & Dravidian & Gondi \\
\hline & & 3. Savaras & 4,148 & 107 & Austro-Asiatic & - \\
\hline & \multirow[t]{4}{*}{ Koraput } & 4. Gadabas & 46,237 & 104 & Austro-Asiatic & - \\
\hline & & 5. Konda Doras & 8,129 & 95 & Dravidian & Kui \\
\hline & & 6. Kuvi Khonds & 325,144 & 100 & Dravidian & Kui \\
\hline & & 7. Parojas & 193,736 & 104 & Dravidian & Parji \\
\hline & \multirow[t]{2}{*}{ Sambalpur } & 8. Binjhals & 50,280 & 104 & Indo-Aryan & - \\
\hline & & 9. Kisans & 87,792 & 108 & Dravidian & - \\
\hline \multirow{5}{*}{$\begin{array}{l}\text { Madhya } \\
\text { Pradesh }\end{array}$} & \multirow[t]{5}{*}{ Bastar } & 10. Bhatras & 71,095 & 105 & Indo-European & Bhatri \\
\hline & & 11. Dhurwas* & - & 86 & Dravidian & Gondi \\
\hline & & 12. Halbas & 57,052 & 97 & Indo-European & Halbi \\
\hline & & 13. Marias* & - & 103 & Dravidian & Gondi \\
\hline & & 14. Murias* & - & 99 & Dravidian & Gondi \\
\hline \multirow[t]{2}{*}{ Maharashtra } & Chandrapur & 15. Maria Gonds & 203,905 & 116 & Dravidian & Gondi \\
\hline & & 16. Raj Gonds & - & 106 & Dravidian & Gondi \\
\hline
\end{tabular}

* Gond group, total no. of population 858,654 . 
Hopkinson et al. (1973) for ESD. Detailed of these techniques are mentioned in Harris and Hopkinson (1976). The G-6-PD deficiency tests were performed in the field itself within a few hours of collection by using brilliant crystal blue dye (BCB) following Motulsky and Campbell-Kraut (1961). The Hb screening was done by cellogel electrophoresis using TEB buffer at $\mathrm{pH} 8.6$ and compared with control samples. All tribes as well as individuals could not be tested for GLO and G-6-PD deficiency due to lack of resources.

\section{RESULTS AND DISCUSSION}

The results of the phenotypic distribution with the Hardy-Weinberg $\chi^{2}$-values and allele frequencies with s.e. of the different markers studied are given in Tables $2-8$.

\section{The distribution}

$A C P$. Genetic polymorphism of red cell enzyme acid phosphatase $(A C P)$ is well known. Table 2 gives the phenotypic and allele frequencies of ACP and it appears that there is genetic equilibrium for all tribal groups except the Raj Gond

Table 2. ACP phenotype frequencies, allele frequencies, and $\chi^{2}$-test for Hardy-Weinberg equilibrium.

\begin{tabular}{|c|c|c|c|c|c|}
\hline \multirow{2}{*}{$\begin{array}{l}\text { Population } \\
\text { (Sample size) }\end{array}$} & \multirow{2}{*}{$\begin{array}{l}\text { Pheno- } \\
\text { type }\end{array}$} & \multicolumn{2}{|c|}{ Total number } & \multirow{2}{*}{$\begin{array}{l}\text { Allele } \\
\text { frequency }\end{array}$} & \multirow{2}{*}{$\begin{array}{l}\chi^{2} \\
(1)\end{array}$} \\
\hline & & & & & \\
\hline Orissa & A & 5 & 5.5 & & \\
\hline \multirow{2}{*}{$\begin{array}{l}\text { Deshia Khonds } \\
(\mathrm{n}=110)\end{array}$} & $\mathrm{AB}$ & 36 & 35.0 & $p^{a}=0.2455$ & \\
\hline & B & 55 & 55.5 & $p^{b}=0.7545$ & 0.499 \\
\hline \multirow{3}{*}{$\begin{array}{l}\text { Raj Gonds } \\
(n=101)\end{array}$} & A & 6 & 4.4 & & \\
\hline & $\mathrm{AB}$ & 30 & 33.3 & $p^{a}=0.2079$ & \\
\hline & $\mathrm{B}$ & 65 & 63.4 & $p^{b}=0.7921$ & 0.974 \\
\hline \multirow{3}{*}{$\begin{array}{l}\text { Savaras } \\
(\mathrm{n}=107)\end{array}$} & A & 5 & 5.4 & & \\
\hline & $\mathrm{AB}$ & 38 & 37.2 & $p^{a}=0.2243$ & \\
\hline & B & 64 & 64.4 & $p^{b}=0.7757$ & 0.045 \\
\hline \multirow{3}{*}{$\begin{array}{l}\text { Gadabas } \\
(n=104)\end{array}$} & A & 4 & 3.3 & & \\
\hline & $A B$ & 29 & 30.4 & $p^{a}=0.1779$ & \\
\hline & B & 71 & 70.3 & $p^{b}=0.8221$ & 0.226 \\
\hline \multirow{3}{*}{$\begin{array}{l}\text { Konda Doras } \\
(n=98)\end{array}$} & $\mathrm{A}$ & 3 & 2.2 & & \\
\hline & $\mathrm{AB}$ & 22 & 23.6 & $p^{a}=0.1573$ & \\
\hline & B & 64 & 63.2 & $p^{b}=0.8427$ & 0.407 \\
\hline \multirow{3}{*}{$\begin{array}{l}\text { Kuvi Khonds } \\
(n=103)\end{array}$} & A & 6 & 4.7 & & \\
\hline & $A B$ & 32 & 34.6 & $p^{a}=0.2136$ & \\
\hline & $\mathrm{B}$ & 65 & 63.7 & $p^{b}=0.7864$ & 0.582 \\
\hline \multirow{3}{*}{$\begin{array}{l}\text { Parojas } \\
(\mathrm{n}=91)\end{array}$} & A & 5 & 4.6 & & \\
\hline & $\mathrm{AB}$ & 31 & 31.8 & $p^{a}=0.2253$ & \\
\hline & B & 55 & 54.6 & $p^{b}=0.7746$ & 0.053 \\
\hline
\end{tabular}


Continued

\begin{tabular}{|c|c|c|c|c|c|}
\hline \multirow{2}{*}{$\begin{array}{l}\text { Population } \\
\text { (Sample size) }\end{array}$} & \multirow{2}{*}{$\begin{array}{l}\text { Pheno- } \\
\text { type }\end{array}$} & \multicolumn{2}{|c|}{ Total number } & \multirow{2}{*}{$\begin{array}{l}\text { Allele } \\
\text { frequency }\end{array}$} & \multirow{2}{*}{$\begin{array}{l}\chi^{2} \\
(1)\end{array}$} \\
\hline & & Obs. & Exp. & & \\
\hline Binjhals & A & 7 & 6.7 & & \\
\hline \multirow[t]{2}{*}{$(n=97)$} & $\mathrm{AB}$ & 37 & 37.6 & $p^{a}=0.2629$ & \\
\hline & $\mathrm{B}$ & 53 & 52.7 & $p^{b}=0.7371$ & 0.024 \\
\hline \multirow{3}{*}{$\begin{array}{l}\text { Kisans } \\
(\mathrm{n}=106)\end{array}$} & A & 4 & 3.6 & & \\
\hline & $\mathrm{AB}$ & 31 & 31.8 & $p^{a}=0.1840$ & \\
\hline & $\mathrm{B}$ & 71 & 70.6 & $p^{b}=0.8160$ & 0.071 \\
\hline \multicolumn{6}{|l|}{ Mahdya Pradesh } \\
\hline Bhatras & A & 6 & 4.4 & & \\
\hline \multirow[t]{2}{*}{$(n=104)$} & $\mathrm{AB}$ & 31 & 34.1 & $p^{a}=0.2067$ & \\
\hline & $\mathrm{B}$ & 67 & 65.5 & $p^{b}=0.7933$ & 0.865 \\
\hline Dhurwas & A & 4 & 3.3 & & \\
\hline \multirow[t]{2}{*}{$(\mathrm{n}=78)$} & $\mathrm{AB}$ & 24 & 25.4 & $p^{a}=0.2051$ & \\
\hline & B & 50 & 49.3 & $p^{b}=0.7949$ & 0.249 \\
\hline Halbas & A & 5 & 5.5 & & \\
\hline \multirow[t]{2}{*}{$(n=96)$} & $\mathrm{AB}$ & 36 & 35.0 & $p^{a}=0.2396$ & \\
\hline & B & 55 & 55.5 & $p^{b}=0.7604$ & 0.082 \\
\hline \multirow{3}{*}{$\begin{array}{l}\text { Marias } \\
(n=101)\end{array}$} & A & 4 & 4.5 & & \\
\hline & $\mathrm{AB}$ & 36 & 34.4 & $p^{a}=0.2178$ & \\
\hline & $\mathrm{B}$ & 61 & 61.8 & $p^{b}=0.7822$ & 0.214 \\
\hline \multirow{3}{*}{$\begin{array}{l}\text { Murias } \\
(\mathrm{n}=105)\end{array}$} & A & 7 & 6.0 & & \\
\hline & $\mathrm{AB}$ & 36 & 38.1 & $p^{a}=0.2381$ & \\
\hline & B & 62 & 60.9 & $p^{\delta}=0.7619$ & 0.318 \\
\hline \multicolumn{6}{|l|}{ Maharashtra } \\
\hline \multirow{3}{*}{$\begin{array}{l}\text { Maria Gonds } \\
(n=115)\end{array}$} & $\mathrm{A}$ & 4 & 6.6 & & \\
\hline & $\mathrm{AB}$ & 47 & 41.9 & $p^{a}=0.2391$ & \\
\hline & $\mathrm{B}$ & 65 & 66.6 & $p^{b}=0.7609$ & 1.743 \\
\hline \multirow{3}{*}{$\begin{array}{l}\text { Raj Gonds } \\
(n=105)\end{array}$} & A & 13 & 8.9 & & \\
\hline & $\mathrm{AB}$ & 35 & 43.3 & $p^{a}=0.2905$ & \\
\hline & B & 57 & 52.9 & $p^{b}=0.7095$ & $3.844^{*}$ \\
\hline
\end{tabular}

* Significant at 5 per cent level.

of Maharashtra. The tribes here show a wide range of variation, the highest frequency for $p^{b}$ allele (0.8427) is found in the Konda Dora of Orissa, and lowest in the Raj Gond of Maharashtra (0.7095). There is no consistent trend of increase or decrease of $p^{b}$ allele frequency across the geographic distances among these tribes but the $p^{b}$ is found to be elevated among few tribes of Kalahandi, Koraput, and Bastar districts. These allele frequencies are in accordance with the frequencies observed in the earlier studies in the tribes of Orissa (Papiha et al., 1988), Madhya Pradesh (Papiha et al., 1978), and Maharashtra (Mukherjee et al., 1979).

$E S D$. Out of all the esterases known in Man only the esterase D (ESD) shows 
Table 3. ESD phenotype frequencies, allele frequencies, and $\chi^{2}$-test for Hardy-Weinberg equilibrium.

\begin{tabular}{|c|c|c|c|c|c|}
\hline \multirow{2}{*}{$\begin{array}{l}\text { Population } \\
\text { (Sample size) }\end{array}$} & \multirow[t]{2}{*}{$\begin{array}{l}\text { Pheno- } \\
\text { type }\end{array}$} & \multicolumn{2}{|c|}{ Total number } & \multirow[t]{2}{*}{$\begin{array}{l}\text { Allele } \\
\text { frequency }\end{array}$} & $\begin{array}{l}\chi^{2} \\
(1)\end{array}$ \\
\hline & & & & & \\
\hline \multirow{3}{*}{$\begin{array}{l}\text { Deshia Khonds } \\
(n=109)\end{array}$} & 1 & 44 & 42.4 & & \\
\hline & $2-1$ & 48 & 51.2 & $E S D^{l}=0.6239$ & \\
\hline & 2 & 17 & 15.4 & $E S D^{2}=0.3716$ & 0.415 \\
\hline \multirow{3}{*}{$\begin{array}{l}\text { Raj Gonds } \\
(n=103)\end{array}$} & 1 & 45 & 44.9 & & \\
\hline & $2-1$ & 46 & 46.2 & $E S D^{1}=0.6602$ & \\
\hline & 2 & 12 & 11.9 & $E S D^{2}=0.3398$ & 0.002 \\
\hline \multirow{3}{*}{$\begin{array}{l}\text { Savaras } \\
(n=107)\end{array}$} & 1 & 40 & 41.9 & & \\
\hline & $2-1$ & 54 & 50.1 & $E S D^{1}=0.6262$ & \\
\hline & 2 & 13 & 14.9 & $E S D^{2}=0.3738$ & 0.651 \\
\hline \multirow{3}{*}{$\begin{array}{l}\text { Gadabas } \\
(n=103)\end{array}$} & 1 & 45 & 48.9 & & \\
\hline & $2-1$ & 52 & 44.1 & $E S D^{1}=0.6893$ & \\
\hline & 2 & 6 & 9.9 & $E S D^{2}=0.3107$ & 3.289 \\
\hline \multirow{3}{*}{$\begin{array}{l}\text { Konda Doras } \\
(n=91)\end{array}$} & 1 & 42 & 41.6 & & \\
\hline & $2-1$ & 39 & 39.9 & $E S D^{1}=0.6758$ & \\
\hline & 2 & 10 & 9.6 & $E S D^{2}=0.3242$ & 0.044 \\
\hline \multirow{3}{*}{$\begin{array}{l}\text { Kuvi Khonds } \\
(n=98)\end{array}$} & 1 & 44 & 44.5 & & \\
\hline & $2-1$ & 44 & 43.1 & $E S D^{1}=0.6735$ & \\
\hline & 2 & 10 & 10.4 & $E S D^{2}=0.3265$ & 0.043 \\
\hline \multirow{3}{*}{$\begin{array}{l}\text { Parojas } \\
(n=89)\end{array}$} & 1 & 31 & 32.2 & & \\
\hline & $2-1$ & 45 & 42.7 & $E S D^{1}=0.6011$ & \\
\hline & 2 & 13 & 14.2 & $E S D^{2}=0.3989$ & 0.263 \\
\hline \multirow{3}{*}{$\begin{array}{l}\text { Binjhals } \\
(\mathrm{n}=100)\end{array}$} & 1 & 35 & 39.1 & & \\
\hline & $2-1$ & 35 & 46.9 & $E S D^{1}=0.6250$ & \\
\hline & 2 & 10 & 14.1 & $E S D^{2}=0.3750$ & 3.004 \\
\hline \multirow{3}{*}{$\begin{array}{l}\text { Kisans } \\
(\mathrm{n}=109)\end{array}$} & 1 & 42 & 41.8 & & \\
\hline & $2-1$ & 51 & 51.4 & $E S D^{1}=0.6193$ & \\
\hline & 2 & 16 & 15.8 & $E S D^{2}=0.3807$ & 0.007 \\
\hline \multicolumn{6}{|l|}{ Madhya Pradesh } \\
\hline & 1 & 42 & 42.5 & & \\
\hline \multirow[t]{2}{*}{$(n=104)$} & $2 \mu 1$ & 49 & 48.0 & $E S D^{1}=0.6394$ & \\
\hline & 2 & 13 & 13.5 & $E S D^{2}=0.3606$ & 0.049 \\
\hline Dhurwas & 1 & 39 & 37.0 & & \\
\hline \multirow[t]{2}{*}{$(\mathrm{n}=76)$} & $2-1$ & 28 & 32.1 & $E S D^{\prime}=0.6974$ & \\
\hline & 2 & 9 & 7.0 & $E S D^{2}=0.3026$ & 1.299 \\
\hline Halbas & 1 & 53 & 51.8 & & \\
\hline \multirow[t]{2}{*}{$(\mathrm{n}=96)$} & $2-1$ & 35 & 37.5 & $E S D^{I}=0.7344$ & \\
\hline & 2 & 8 & 6.8 & $E S D^{2}=0.2656$ & 0.412 \\
\hline
\end{tabular}


Continued

\begin{tabular}{|c|c|c|c|c|c|}
\hline \multirow{2}{*}{$\begin{array}{l}\text { Population } \\
\text { (Sample size) }\end{array}$} & \multirow{2}{*}{$\begin{array}{l}\text { Pheno- } \\
\text { type }\end{array}$} & \multicolumn{2}{|c|}{ Total number } & \multirow{2}{*}{$\begin{array}{l}\text { Allele } \\
\text { frequency }\end{array}$} & \multirow{2}{*}{$\begin{array}{l}\chi^{2} \\
(1)\end{array}$} \\
\hline & & Obs. & Exp. & & \\
\hline Marias & 1 & 59 & 57.4 & & \\
\hline \multirow[t]{2}{*}{$(n=102)$} & $2-1$ & 35 & 38.2 & $E S D^{1}=0.7500$ & \\
\hline & 2 & 8 & 6.4 & $E S D^{2}=0.2500$ & 0.736 \\
\hline Murias & 1 & 43 & 44.2 & & \\
\hline \multirow[t]{2}{*}{$(n=103)$} & $2-1$ & 49 & 46.5 & $E S D^{I}=0.6553$ & \\
\hline & 2 & 11 & 12.2 & $E S D^{2}=0.3447$ & 0.290 \\
\hline \multicolumn{6}{|l|}{ Maharashtra } \\
\hline Maria Gonds & 1 & 46 & 46.8 & & \\
\hline \multirow[t]{2}{*}{$(n=93)$} & $2-1$ & 40 & 38.3 & $E S D^{I}=0.7097$ & \\
\hline & 2 & 7 & 7.8 & $E S D^{2}=0.2903$ & 0.178 \\
\hline Raj Gonds & 1 & 56 & 54.8 & & \\
\hline \multirow[t]{2}{*}{$(n=96)$} & $2-1$ & 33 & 35.5 & $E S D^{I}=0.7552$ & \\
\hline & 2 & 7 & 5.8 & $E S D^{2}=0.2448$ & 0.474 \\
\hline
\end{tabular}

polymorphism on the basis on two common alleles $E S D^{1}$ and $E S D^{2}$, besides a few rare alleles. The tribes understudy show a complete agreement with the HardyWeinberg equilibrium except among two tribes the Gadaba and the Binjhal where homozygotes exceed in number, and three common phenotypes 1-1, 2-1, 2-2 are present. Table 3 shows a variation in allele frequencies. The $E S D^{2}$ allele frequency ranges between 0.2448 in the Raj Gond of Maharashtra and 0.3989 in the Paroja of Orissa. The present allele frequencies of $E S D^{2}$ remain within the range of reported allele frequencies among Indian tribes which show a wide range of variation, that is lowest $E S D^{2}(0.022)$ among the Gaddi Rajput of Himachal Pradesh (Singh et al., 1982) and a highest (0.475) is in the Kolam of Andhra Pradesh (Ramesh et al., 1979). In general, there has been a gradual decline of $E S D^{2}$ allele from east to west direction except for a few transient elevation among the Bhatra of Bastar and among the Paroja of Koraput districts.

$P G D$. 6-Phosphogluconate dehydrogenase (6-PGD) shows usually three phenotypes controlled by two alleles $P G D^{A}$ and $P G D^{C}$. In regard to $P G D$ alleles genetic equilibrium has been observed among almost all the tribes (Table 4). In a few cases when significant $\chi^{2}$-values have been obtained it seems not to be much meaningful because of small number of $P G D c$ allele. Out of the 16 tribes studied the $P G D^{A}$ appears to be monomorphic in three tribes namely Deshia Khonds, Konda Doras, and Kisans of Orissa and in rest of the populations both $P G D^{A}$ and $P G D^{C}$ are present, having maximum $P G D^{c}$ frequency in the Bhatra $(0.160)$ of Bastar of Madhya Pradesh. Highest frequency of $P G D^{C}$ was reported among the Kadar (0.167) of Tamilnadu (Saha et al., 1974), and in many of the other tribes this allele is found to be absent. The $P G D^{A}$ allele frequency remains to be more or less similar 
Table 4. PGD phenotype frequencies, allele frequencies, and $\chi^{2}$-test for Hardy-Weinberg equilibrium.

\begin{tabular}{|c|c|c|c|c|c|}
\hline \multirow{2}{*}{$\begin{array}{l}\text { Population } \\
\text { (Sample size) }\end{array}$} & \multirow{2}{*}{$\begin{array}{l}\text { Pheno- } \\
\text { type }\end{array}$} & \multicolumn{2}{|c|}{ Total number } & \multirow{2}{*}{$\begin{array}{l}\text { Allele } \\
\text { frequency }\end{array}$} & \multirow{2}{*}{$\begin{array}{l}\chi^{2} \\
(1)\end{array}$} \\
\hline & & Obs. & Exp. & & \\
\hline \multicolumn{6}{|l|}{ Orissa } \\
\hline Deshia Khonds & A & 108 & 108.0 & & \\
\hline \multirow[t]{2}{*}{$(\mathrm{n}=108)$} & $\mathrm{AC}$ & - & - & $P G D^{A}=1.0000$ & \\
\hline & C & - & - & $P G D C=-$ & - \\
\hline Raj Gonds & A & 98 & 98.0 & & \\
\hline \multirow[t]{2}{*}{$(\mathrm{n}=99)$} & $\mathrm{AC}$ & 1 & 1.0 & $P G D A=0.9949$ & \\
\hline & $\mathrm{C}$ & - & 0.1 & $P G D^{C}=0.0051$ & - \\
\hline Savaras & A & 104 & 104.0 & & \\
\hline \multirow[t]{2}{*}{$(\mathrm{n}=105)$} & $\mathrm{AC}$ & 1 & 1.0 & $P G D A=0.9952$ & \\
\hline & $\mathrm{C}$ & - & 0.1 & $P G D^{C}=0.0048$ & - \\
\hline Gadabas & A & 102 & 102.0 & & \\
\hline \multirow[t]{2}{*}{$(n=104)$} & $\mathrm{AC}$ & 2 & 2.0 & $P G D^{A}=0.9904$ & \\
\hline & $\mathrm{C}$ & - & 0.1 & $P G D C=0.0096$ & - \\
\hline Konda Doras & A & 85 & 85.0 & & \\
\hline \multirow[t]{2}{*}{$(\mathrm{n}=85)$} & $\mathrm{AC}$ & - & - & $P G D A=1.0000$ & \\
\hline & $\mathrm{C}$ & - & - & $P G D C=-$ & - \\
\hline Kuvi Khonds & A & 100 & 99.0 & & \\
\hline \multirow[t]{2}{*}{$(\mathrm{n}=102)$} & $\mathrm{AC}$ & 1 & 3.0 & $P G D^{A}=0.9853$ & \\
\hline & $\mathrm{C}$ & 1 & 0.1 & $P G D C=0.0147$ & \\
\hline Parojas & $A$ & 90 & 90.0 & & \\
\hline \multirow[t]{2}{*}{$(n=92)$} & $\mathrm{AC}$ & 2 & 2.0 & $P G D^{A}=0.9891$ & \\
\hline & $\mathrm{C}$ & - & 0.1 & $F G D C=0.1009$ & - \\
\hline Binjhals & A & 86 & 86.2 & & \\
\hline \multirow[t]{2}{*}{$(\mathrm{n}=94)$} & $\mathrm{AC}$ & 8 & 7.7 & $P G D^{A}=0.9574$ & \\
\hline & $\mathrm{C}$ & - & 0.2 & $P G D^{C}=0.0426$ & 0.186 \\
\hline Kisans & A & 106 & 106.0 & & \\
\hline \multirow[t]{2}{*}{$(\mathrm{n}=106)$} & $\mathrm{AC}$ & - & - & $P G D^{A}=1.0000$ & \\
\hline & $\mathrm{C}$ & - & - & $P G D^{C}=-$ & - \\
\hline \multicolumn{6}{|l|}{ Madhya Pradesh } \\
\hline Bhatras & A & 66 & 66.5 & & \\
\hline \multirow[t]{2}{*}{$(n=97)$} & $\mathrm{AC}$ & 31 & 26.1 & $P G D^{A}=0.8402$ & \\
\hline & $\mathrm{C}$ & - & 2.5 & $P G D^{C}=0.1598$ & 3.508 \\
\hline \multirow{3}{*}{$\begin{array}{l}\text { Dhurwas } \\
(\mathrm{n}=78)\end{array}$} & $\mathrm{A}$ & 72 & 72.1 & & \\
\hline & $\mathrm{AC}$ & 6 & 5.8 & $P G D^{A}=0.9615$ & \\
\hline & $\mathrm{C}$ & - & 0.1 & $P G D^{C}=0.0385$ & 0.125 \\
\hline Halbas & A & 74 & 74.0 & & \\
\hline \multirow[t]{2}{*}{$(\mathrm{n}=75)$} & $\mathrm{AC}$ & 1 & 1.0 & $P G D^{A}=0.9933$ & \\
\hline & $\mathrm{C}$ & - & 0.1 & $P G D^{C}=0.0067$ & - \\
\hline Marias & A & 82 & 82.2 & & \\
\hline \multirow[t]{2}{*}{$(n=91)$} & $\mathrm{AC}$ & 9 & 8.6 & $P G D A=0.9505$ & \\
\hline & $\mathrm{C}$ & - & 0.2 & $P G D^{C}=0.0495$ & 0.246 \\
\hline
\end{tabular}




\begin{tabular}{|c|c|c|c|c|c|}
\hline \multirow{2}{*}{$\begin{array}{l}\text { Population } \\
\text { (Sample size) }\end{array}$} & \multirow{2}{*}{$\begin{array}{l}\text { Pheno- } \\
\text { type }\end{array}$} & \multicolumn{2}{|c|}{ Total number } & \multirow{2}{*}{$\begin{array}{l}\text { Allele } \\
\text { frequency }\end{array}$} & \multirow{2}{*}{$\begin{array}{l}z^{2} \\
(1)\end{array}$} \\
\hline & & Obs. & Exp. & & \\
\hline Murias & $\mathrm{A}$ & 93 & 93.0 & & \\
\hline \multirow[t]{2}{*}{$(n=96)$} & $\mathrm{AC}$ & 3 & 3.0 & $P G D^{A}=0.9844$ & \\
\hline & $\mathrm{C}$ & - & 0.1 & $P G D^{C}=0.0156$ & - \\
\hline \multicolumn{6}{|l|}{ Maharashtra } \\
\hline Maria Gonds & A & 100 & 98.7 & & \\
\hline \multirow[t]{2}{*}{$(\mathrm{n}=116)$} & $\mathrm{AC}$ & 14 & 16.6 & $P G D^{A}=0.9224$ & \\
\hline & $\mathrm{C}$ & 2 & 0.7 & $P G D^{C}=0.776$ & 2.852 \\
\hline Raj Gonds & $\mathrm{A}$ & 101 & 101.1 & & \\
\hline \multirow[t]{2}{*}{$(n=106)$} & $\mathrm{AC}$ & 5 & 4.9 & $P G D^{A}=0.9764$ & \\
\hline & $\mathrm{C}$ & - & 0.1 & $P G D^{C}=0.0236$ & 0.062 \\
\hline
\end{tabular}

among the population of Orissa and then gradually declines towards west.

GLO. Glyoxalase (GLO) marker could be tested only in 9 tribes, and the phenotypic and allele frequencies are given in Table 5. There are two common alleles $G L O^{1}$ and $G L O^{2}$ and both occur frequently in these populations, of which $G L O^{2}$ is more predominant. Hardy-Weinberg equilibrium test shows a statistically significant deviation in case of Gadabas and Parojas of Orissa, and among the Maria Gond of Maharashtra. In this study $G L O^{1}$ shows maximum frequency $(0.422)$ in Maria Gonds of Maharashtra (the maximum value present in Marias of Madhya Pradesh but is ignored as the sample size is very small) and minimum (0.263) in Parojas of Orissa. No cline has been observed in regard to GLO allele frequency distribution.

G-6-PD deficiency. Glucose-6-phosphate dehydrogenase (G-6-PD) deficiency is a well known X-linked trait. Only 319 male individuals of the five tribes were tested, which are shown in the Table 6. The samples from other tribes could not be tested for the lack of resources. In the present series the incidence of the deficiency have been noticed only in two tribes namely Muria and Bhatra of Madhya Pradesh, the frequencies being 2.74 and $2.94 \%$ respectively. In some Indian tribes, tested so far, the deficiency rate is as high as $19.62 \%$ in Worli of Nagar Dadar Haviely (Joshi et al., 1975). Amongst the Gond tribe of Nagpur, Maharashtra, and Raipur of Madhya Pradesh the deficiencies are 10.58\% (Kher et al., 1967) and 15.08\% respectively (Report of Institute of Immunohaematology, ICMR, Bombay, 1986). The deficiency trait is recognised even in many non-tribal populations in India, and it is high amongst the Parsis (17.30\%) of Bombay (Kate et al., 1978). The high incidence of G-6-PD deficiency and some abnormal haemoglobins in malarial environment is a well known fact. It has also been suggested that the protection is conferred against malarial parasite by the heterozygous females (Bienzle et al., 1972; Kar et al., 1992) which could be extended to hemizygous also. 
Table 5. GLO phenotype frequencies, allele frequencies, and $x^{2}$-text for Hardy-Weinberg equilibrium.

\begin{tabular}{|c|c|c|c|c|c|}
\hline \multirow{2}{*}{$\begin{array}{l}\text { Population } \\
\text { (Sample size) }\end{array}$} & \multirow{2}{*}{$\begin{array}{l}\text { Pheno- } \\
\text { type }\end{array}$} & \multicolumn{2}{|c|}{ Total number } & \multirow{2}{*}{$\begin{array}{l}\text { Allele } \\
\text { frequency }\end{array}$} & \multirow{2}{*}{$\begin{array}{l}\chi^{2} \\
(1)\end{array}$} \\
\hline & & Obs. & Exp. & & \\
\hline \multicolumn{6}{|l|}{ Orissa } \\
\hline Gadabas & 1 & 12 & 7.5 & & \\
\hline \multirow[t]{2}{*}{$(\mathrm{n}=71)$} & $2-1$ & 22 & 31.1 & $G L O 1=0.3239$ & \\
\hline & 2 & 37 & 32.5 & $G L O^{2}=0.6761$ & $6.078^{*}$ \\
\hline \multirow{3}{*}{$\begin{array}{l}\text { Konda Doras } \\
(\mathrm{n}=28)\end{array}$} & 1 & 6 & 4.3 & & \\
\hline & $2-1$ & 10 & 13.4 & $G L O^{1}=0.3928$ & \\
\hline & 2 & 12 & 10.3 & $G L O^{2}=0.6071$ & 1.769 \\
\hline \multirow{3}{*}{$\begin{array}{l}\text { Parojas } \\
(\mathrm{n}=93)\end{array}$} & 1 & 12 & 6.5 & & \\
\hline & $2-1$ & 25 & 36.1 & $G L O I=0.2634$ & \\
\hline & 2 & 56 & 50.5 & $G L O^{2}=0.7366$ & $8.783^{* *}$ \\
\hline \multicolumn{6}{|l|}{ Madhya Pradesh } \\
\hline Bhatras & 1 & 11 & 15.0 & & \\
\hline \multirow[t]{2}{*}{$(\mathrm{n}=104)$} & $2-1$ & 57 & 49.0 & $G L O I=03798$ & \\
\hline & 2 & 36 & 40.0 & $G L O^{2}=0.6202$ & 2.776 \\
\hline Halbas & 1 & 14 & 11.6 & & \\
\hline \multirow[t]{2}{*}{$(\mathrm{n}=97)$} & $2-1$ & 39 & 43.9 & $G L O^{I}=0.3454$ & \\
\hline & 2 & 44 & 41.6 & $G L O^{2}=0.6546$ & 1.191 \\
\hline Marias & 1 & 4 & 3.5 & & \\
\hline \multirow[t]{2}{*}{$(n=14)$} & $2-1$ & 6 & 7.0 & $G L O^{1}=0.5000$ & \\
\hline & 2 & 4 & 3.5 & $G L O^{2}=0.5000$ & 0.286 \\
\hline \multirow{3}{*}{$\begin{array}{l}\text { Murias } \\
(n=66)\end{array}$} & 1 & 9 & 8.7 & & \\
\hline & $2-1$ & 30 & 30.6 & $G L O 1=0.3636$ & \\
\hline & 2 & 27 & 26.7 & $G L O^{2}=0.6364$ & 0.021 \\
\hline \multicolumn{6}{|l|}{ Maharashtra } \\
\hline \multirow{3}{*}{$\begin{array}{l}\text { Maria Gonds } \\
(\mathrm{n}=45)\end{array}$} & 1 & 13 & 8.0 & & \\
\hline & $2-1$ & 12 & 22.0 & $G L O^{1}=0.4222$ & \\
\hline & 2 & 20 & 15.0 & $G L O^{2}=0.5778$ & $9.252^{* *}$ \\
\hline \multirow{3}{*}{$\begin{array}{l}\text { Raj Gonds } \\
(\mathrm{n}=40)\end{array}$} & $!$ & 5 & 3.3 & & \\
\hline & $2-1$ & 13 & 16.4 & $G L O^{1}=0.2875$ & \\
\hline & 2 & 22 & 20.3 & $G L O^{2}=0.7125$ & 1.709 \\
\hline
\end{tabular}

* Significant at 5 per cent level; ** at 1 per cent level.

Haemoglobin variants. Study of haemoglobin pattern was done in all the tribal groups. The phenotypic and gene distribution are shown in the Table 7. Two major variant haemoglobin types $\mathrm{HbE}$ and $\mathrm{HbS}$ are common in some Indian populations, of which $\mathrm{HbE}$ is mostly restricted among the populations of Eastern and North-Eastern India, whereas HbS is dominant in South, West, and Central India. In the present study $\mathrm{HbS}$ is found in all the tribes with varying frequencies, 
Table 6. G-6-PD deficiency distribution.

\begin{tabular}{llr}
\hline $\begin{array}{l}\text { Population } \\
\text { (Sample size) }\end{array}$ & $\begin{array}{l}\text { Normal/ } \\
\text { deficient }\end{array}$ & $\begin{array}{c}\text { Total } \\
\text { observed No. }\end{array}$ \\
\hline Madhya Pradesh & Normal & 102 \\
Bhatra & $\mathrm{n}=105)$ & Deficient \\
Dhurwa & Normal & 3 \\
$(\mathrm{n}=23)$ & Deficient & 23 \\
Halba & Normal & 0 \\
$(\mathrm{n}=69)$ & Deficient & 69 \\
Maria & Normal & 0 \\
$(\mathrm{n}=47)$ & Deficient & 47 \\
Muria & Normal & 0 \\
$(\mathrm{n}=75)$ & Deficient & 73 \\
\hline
\end{tabular}

Table 7. Haemoglobin phenotype frequencies, allele frequencies, and $\chi^{2}$-test for HardyWeinberg equilibrium.

\begin{tabular}{|c|c|c|c|c|c|}
\hline \multirow{2}{*}{$\begin{array}{l}\text { Population } \\
\text { (Sample size) }\end{array}$} & \multirow{2}{*}{$\begin{array}{l}\text { Pheno- } \\
\text { type }\end{array}$} & \multicolumn{2}{|c|}{ Total number } & \multirow{2}{*}{$\begin{array}{l}\text { Allele } \\
\text { frequency }\end{array}$} & \multirow{2}{*}{$\begin{array}{l}\chi^{2} \\
(1)\end{array}$} \\
\hline & & Obs. & Exp. & & \\
\hline \multicolumn{6}{|l|}{ Orissa } \\
\hline \multirow{3}{*}{$\begin{array}{l}\text { Deshia Khonds } \\
(\mathrm{n}=107)\end{array}$} & A & 98 & 98.2 & & \\
\hline & AS & 9 & 8.6 & $H b^{A}=0.9579$ & \\
\hline & $\mathrm{S}$ & - & 0.2 & $H b^{S}=0.0421$ & 0.206 \\
\hline \multirow{3}{*}{$\begin{array}{l}\text { Raj Gonds } \\
(\mathrm{n}=104)\end{array}$} & A & 98 & 98.1 & & \\
\hline & AS & 6 & 5.8 & $H b^{A}=0.9712$ & \\
\hline & $\mathrm{s}$ & - & 0.1 & $H b^{S}=0.0288$ & 0.092 \\
\hline \multirow{3}{*}{$\begin{array}{l}\text { Savaras } \\
(n=102)\end{array}$} & A & 102 & 102.0 & & \\
\hline & AS & - & - & $H b^{A}=1.0000$ & \\
\hline & $\mathrm{S}$ & - & - & $H b s=0.0000$ & - \\
\hline \multirow{3}{*}{$\begin{array}{l}\text { Gadabas } \\
(\mathrm{n}=104)\end{array}$} & A & 95 & 94.2 & & \\
\hline & AS & 8 & 9.5 & $H b^{A}=0.9519$ & \\
\hline & $\mathrm{s}$ & 1 & 0.2 & $H b^{S}=0.0481$ & 2.649 \\
\hline \multirow{3}{*}{$\begin{array}{l}\text { Konda Doras } \\
(n=86)\end{array}$} & A & 72 & 71.7 & & \\
\hline & AS & 13 & 13.7 & $H b^{A}=0.9128$ & \\
\hline & $\mathrm{S}$ & 1 & 0.6 & $H b S=0.0872$ & 0.220 \\
\hline \multirow{3}{*}{$\begin{array}{l}\text { Kuvi Khonds } \\
(n=94)\end{array}$} & A & 91 & 90.0 & & \\
\hline & AS & 2 & 3.9 & $H b^{A}=0.9787$ & \\
\hline & $\mathrm{s}$ & 1 & 0.1 & $H b S=0.0213$ & $22.489^{* * *}$ \\
\hline \multirow{3}{*}{$(\mathrm{n}=94)$} & A & 86 & 84.3 & & \\
\hline & AS & 6 & 9.5 & $H b^{A}=0.9468$ & \\
\hline & $\mathrm{S}$ & 2 & 0.3 & $H b S=0.0532$ & $12.612 * * *$ \\
\hline
\end{tabular}

Vol. 38, No, 3, 1993 
Continued

\begin{tabular}{|c|c|c|c|c|c|}
\hline \multirow{2}{*}{$\begin{array}{l}\text { Population } \\
\text { (Sample size) }\end{array}$} & \multirow{2}{*}{$\begin{array}{l}\text { Pheno- } \\
\text { type }\end{array}$} & \multicolumn{2}{|c|}{ Total number } & \multirow{2}{*}{$\begin{array}{l}\text { Allele } \\
\text { frequency }\end{array}$} & \multirow{2}{*}{$\begin{array}{l}x^{2} \\
(1)\end{array}$} \\
\hline & & Obs. & Exp. & & \\
\hline Binjhals & A & 93 & 90.4 & & \\
\hline \multirow[t]{2}{*}{$(n=103)$} & AS & 7 & 12.2 & $H b^{A}=0.9369$ & \\
\hline & $\mathrm{S}$ & 3 & 0.4 & $H b^{S}=0.0631$ & $18.628^{* * *}$ \\
\hline Kisans & A & 104 & 101.1 & & \\
\hline \multirow[t]{2}{*}{$(\mathrm{n}=108)$} & AS & 1 & 6.8 & $H b^{A}=0.9676$ & \\
\hline & $\mathrm{S}$ & 3 & 0.1 & $H b^{s}=0.0324$ & $78.464^{* * *}$ \\
\hline \multicolumn{6}{|l|}{ Madhya Pradesh } \\
\hline Bhatras & A & 90 & 88.5 & & \\
\hline \multirow[t]{2}{*}{$(n=102)$} & AS & 10 & 13.0 & $H b^{A}=0.9314$ & \\
\hline & $S$ & 2 & 0.5 & $H b^{S}=0.0686$ & $5.541 *$ \\
\hline Dhurwas & A & 74 & 72.3 & & \\
\hline \multirow{2}{*}{$(n=81)$} & AS & 5 & 8.5 & $H b^{A}=0.9444$ & \\
\hline & 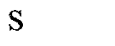 & 2 & 0.3 & $H b^{S}=0.0556$ & $13.730^{* * *}$ \\
\hline Halbas & A & 85 & 83.7 & & \\
\hline \multirow[t]{2}{*}{$(n=99)$} & AS & 12 & 14.7 & $H b^{A}=0.9192$ & \\
\hline & $\mathrm{S}$ & 2 & 0.7 & $H b^{S}=0.0808$ & 3.354 \\
\hline Marias & A & 73 & 72.4 & & \\
\hline \multirow[t]{2}{*}{$(\mathrm{n}=94)$} & AS & 19 & 20.2 & $H b^{A}=0.8777$ & \\
\hline & $S$ & 2 & 1.4 & $H b S=0.1223$ & 0.325 \\
\hline Murias & A & 85 & 84.7 & & \\
\hline \multirow[t]{2}{*}{$(n=101)$} & AS & 15 & 15.6 & $H b^{A}=0.9158$ & \\
\hline & $S$ & 1 & 0.7 & $H b^{S}=0.0842$ & 0.135 \\
\hline \multicolumn{6}{|l|}{ Maharashtra } \\
\hline Maria Gonds & $\mathrm{A}$ & 85 & 84.1 & & \\
\hline \multirow[t]{2}{*}{$(n=113)$} & AS & 25 & 26.8 & $H b^{A}=0.8628$ & \\
\hline & $\mathrm{S}$ & 3 & 2.1 & $H b^{S}=0.1372$ & 0.482 \\
\hline Raj Gonds & A & 92 & 91.3 & & \\
\hline \multirow[t]{2}{*}{$(n=102)$} & AS & 9 & 10.4 & $H b^{A}=0.9461$ & \\
\hline & $s$ & 1 & 0.3 & $H b S=0.0539$ & 1.864 \\
\hline
\end{tabular}

* Significant at 5 per cent level; *** at 0.1 per cent level.

except Savaras of Orissa. HbS in homozygous form is present, surprisingly, in all the tribes except three tribes-Dashia Khonds, Raj Gonds, and Savaras of Orissa. The maximum number of cases (three cases) have been observed in the Kisans of Orissa. The highest HbS allele frequency $(0.1372)$ is found amongst the Maria Gond. It is noteworthy that a few of the HbSS types were detected among the adult and old individuals also. The genetic equilibrium can be assumed for a majority of the groups, though significant deviations are noticed in six of them: 
Kuvi Khonds, Paroja, Binjhal, and Kisan of Orissa and Bhatra and Dhurwa of Madhya Pradesh. The genetic equilibrium obtained through $\chi^{2}$-tests may not be that appropriate because in majority cases HbS homozygous number seems to be inadequate. A geographical cline of $H b S$ allele increasing from east to west has been observed. A large number of Indian populations including tribes have been screened for $\mathrm{Hb}$ type (Mukherjee and Das, 1990). The presence of several HbSS cases in this region raises the question as to how it is maintained in a high frequencies despite its known lethality in homozygous condition. One of the reasons may be cooccurrence of $\alpha$-thalassemia along with $\mathrm{HbS}$ which reduces the lethality of $\mathrm{HbS}$, and increased level of $\mathrm{HbF}$ among the $\mathrm{HbS}$ heterozygous or homozygous individuals. High frequencies (about $16 \%$ ) of $S$ allele have been reported amongst the Gamit of Gujrat, Western India (Vyas et al., 1962) and in the Bhilala tribes of Madhya Pradesh (ICMR report, 1986); about $13 \%$ in the Konda Paroja or Orissa (Das et al., 1967), and in South India it is also high (about $18 \%$ ) amongst the Pradhan of Andhra Pradesh (Blake et al., 1981).

$L D H$. Lactate dehydrogenase is normally monomorphic in world populations. In India, the LDH Cal-1 variant is found to be widely distributed. All the 16 tribes were screened for LDH type and shown in Table 8. LDH Cal-1 variant was detected in many of these tribes, except Savaras, Parojas, Binjhals, Kisans, Halbas, and Raj Gonds (Maharashtra). It is worth mentioning that two cases of Cal-1 homozygous variant is usually rare, were detected in two tribes of Orissa, namely in Deshia Khonds and in Raj Gonds. Maximum number (five cases) of Cal-1 variant were identified amongst the Kuvi Khonds of Orissa. LDH Cal allele is relatively more frequent in the Hindu caste groups rather than tribes in

Table 8. LDH phenotype frequencies and allele frequencies.

\begin{tabular}{|c|c|c|c|c|}
\hline $\begin{array}{l}\text { Population } \\
\text { (Sample size) }\end{array}$ & Phenotype & $\begin{array}{l}\text { Total } \\
\text { Obs. }\end{array}$ & \multicolumn{2}{|c|}{ Allele frequency } \\
\hline \multicolumn{5}{|l|}{ Orissa } \\
\hline Deshia Khonds & $\mathbf{N}$ & 103 & $L D H^{N}$ & 0.9811 \\
\hline \multirow[t]{2}{*}{$(\mathrm{n}=106)$} & CAL 1 & 2 & $L D H C a l 1$ & 0.0189 \\
\hline & CAL 1 (Homo) & 1 & & \\
\hline Raj Gonds & $\mathrm{N}$ & 104 & $L D H^{N}$ & 0.9859 \\
\hline \multirow[t]{2}{*}{$(\mathrm{n}=106)$} & CAL 1 & 1 & LDHCal 1 & 0.0141 \\
\hline & CAL 1 (Homo) & 1 & & \\
\hline Savaras & $\mathrm{N}$ & 105 & $L D H^{N}$ & 1.0000 \\
\hline \multirow[t]{2}{*}{$(n=105)$} & CAL 1 & - & $L D H C a l 1$ & 0.0000 \\
\hline & CAL 1 (Homo) & - & & \\
\hline Gadabas & $\mathrm{N}$ & 94 & $L D H^{N}$ & 0.9947 \\
\hline \multirow[t]{2}{*}{$(\mathrm{n}=95)$} & CAL 1 & 1 & $L D H C a l 1$ & 0.0053 \\
\hline & CAL 1 (Homo) & - & & \\
\hline
\end{tabular}

Vol. 38, No. 3, 1993 
Continued

\begin{tabular}{|c|c|c|c|c|}
\hline \multirow{4}{*}{$\begin{array}{l}\begin{array}{l}\text { Population } \\
\text { (Sample size) }\end{array} \\
\begin{array}{l}\text { Konda Doras } \\
(\mathrm{n}=88)\end{array}\end{array}$} & \multirow{2}{*}{ Phenotype } & \multirow{2}{*}{$\begin{array}{l}\text { Total } \\
\text { Obs. }\end{array}$} & \multicolumn{2}{|c|}{ Allele frequency } \\
\hline & & & $L D H^{A}$ & 0.9943 \\
\hline & CAL 1 & 1 & $L D H C a l I$ & 0.0057 \\
\hline & CAL I (Homo) & - & & \\
\hline Kuvi Khonds & $N$ & 93 & $L D H^{A}$ & 0.9745 \\
\hline \multirow[t]{2}{*}{$(n=98)$} & CAL 1 & 5 & $L D H C a l 1$ & 0.0255 \\
\hline & CAL I (Homo) & - & & \\
\hline Parojas & $\mathbf{N}$ & 105 & $L D H^{A}$ & 1.0000 \\
\hline \multirow[t]{2}{*}{$(n=105)$} & CAL 1 & - & $L D H C a l l$ & 0.0000 \\
\hline & CAL 1 (Homo) & $\cdots$ & & \\
\hline Binjhals & $N$ & 102 & $L D H^{A}$ & 1.0000 \\
\hline \multirow[t]{2}{*}{$(n=102)$} & CAL 1 & - & LDHCal L. & 0.0000 \\
\hline & CAL I (Homo) & - & & \\
\hline Kisans & N & 103 & $L D H^{A}$ & 1.0000 \\
\hline \multirow[t]{2}{*}{$(n=103)$} & CAL! & - & LDHCal I & 0.0000 \\
\hline & CAL 1 (Homo) & - & & \\
\hline \multicolumn{5}{|l|}{ Maharashtra } \\
\hline Bhatrass & $N$ & 102 & $L D H^{A}$ & 0.9951 \\
\hline \multirow[t]{2}{*}{$(n=103)$} & CAL: & 1 & LDHCal I & 0.0049 \\
\hline & CAL 1 (Homo) & - & & \\
\hline Dhurwas & $\mathrm{N}$ & 81 & $L D H A$ & 0.9939 \\
\hline \multirow[t]{2}{*}{$(\mathrm{n}=82)$} & CAL & 1 & $L D H C a l 1$ & 0.0061 \\
\hline & CAL 1 (Homo) & - & & \\
\hline Halbas & $N$ & 94 & $L D H^{A}$ & 1.0000 \\
\hline \multirow[t]{2}{*}{$(n=94)$} & CAL 1 & - & LDHCal 1 & 0.0000 \\
\hline & CAL 1 (Homo) & - & & \\
\hline Marias & $\mathrm{N}$ & 98 & $L D H^{A}$ & 0.9949 \\
\hline \multirow[t]{2}{*}{$(n=99)$} & CAL 1 & 1 & LDHCal I & 0.0051 \\
\hline & CAL 1 (Homo) & -- & & \\
\hline Murias & $\mathrm{N}$ & 105 & $\angle O H A$ & 0.9953 \\
\hline \multirow{2}{*}{$(\mathrm{n}=106)$} & CAL 1 & 1 & LDHCal I & 0.0047 \\
\hline & CAL 1 (Homo) & - & & \\
\hline \multicolumn{5}{|l|}{ Maharashtra } \\
\hline Maria Gonds & $\mathrm{N}$ & 115 & $L D H^{A}$ & 0.9957 \\
\hline \multirow[t]{2}{*}{$(n=116)$} & CAL & 1 & LDHCal 1 & 0.0043 \\
\hline & CAL 1 (Homo) & $\ldots$ & & \\
\hline Raj Gonds & $\mathrm{N}$ & 107 & $L D H^{A}$ & 1.0000 \\
\hline \multirow{2}{*}{$(n=107)$} & CAL 1 & - & LDHCal 1 & 0.0000 \\
\hline & CAL I (Homo) & -. & & \\
\hline
\end{tabular}


Table 9. Analysis of heterogeneity in 16 tribal groups of Orissa, Madhya Pradesh, and Maharashtra, based on four enzyme and haemoglobin loci.

\begin{tabular}{|c|c|c|c|c|c|c|c|c|}
\hline Locus & Allele & $\begin{array}{l}\text { No. of } \\
\text { popula- } \\
\text { tion }\end{array}$ & $\begin{array}{l}\text { No. of } \\
\text { individ- } \\
\text { ual } \\
\text { tested }\end{array}$ & $x^{2}$ & d.f & $\mathrm{H}_{\mathrm{T}}$ & $\mathrm{G}_{\mathrm{ST}}$ & DST $_{\text {ST }}$ \\
\hline$A C P$ & $p^{\alpha}$ & 16 & 1,611 & 2.5785 & 15 & 0.3443 & 0.0058 & 0.0020 \\
\hline$E S D$ & $E S D^{1}$ & 16 & 1,587 & 0.0227 & 15 & 0.4415 & 0.0100 & 0.0044 \\
\hline $6-P G D$ & $P G D^{A}$ & 16 & 1,554 & 0.0140 & 15 & 0.0558 & 0.0574 & 0.0032 \\
\hline$G L O$ & $G L O^{1}$ & 9 & 772 & 11.0755 & 15 & - & - & - \\
\hline$H b$ & $H b^{S}$ & 16 & 1,492 & 1.2408 & 15 & 0.1221 & 0.0164 & 0.0020 \\
\hline
\end{tabular}

India (Mukherjee and Reddy, 1983). Highest frequency of Cal-1 type has been reported among the Kurumbas of Nilgiri Hill (Saha et al., 1976).

Gene diversity

Table 9 provides $\chi^{2}$-values for testing the homogeneity of gene frequencies as well as the $\mathrm{G}_{\mathrm{ST}}$ values. The $\chi^{2}$-values indicate a non-significant heterogeneity for all alleles in the individual systems. For measuring the extent of gene diversity $\mathrm{G}_{\mathrm{ST}}$ computation was performed using all 16 populations together and shown in the same Table 9. The total gene diversity $\left(\mathrm{H}_{\mathrm{T}}\right)$ of the tribes appears to be 0.3678 , while the average gene diversity within populations $\left(\mathrm{H}_{\mathrm{S}}\right)$ is 0.4474 and between populations $\left(\mathrm{D}_{\mathrm{ST}}\right)$ is found to be 0.0044 . Within population diversity is larger than between population diversity. The $\mathrm{G}_{\mathrm{ST}}$ value is smallest $(0.0020)$ for $p a$ allele and highest for $H b^{s}$ gene $(0.0415)$. The degree of over all genetic differentiation as measured by the total $G_{\mathrm{ST}}$ values is $0.0154 \pm 0.0071$ which seems to be statistically non significant. The sporadic trends of geographical cline observed in the distribution of afore mentioned markers do not, in fact, offer any meaningful explanation for common origin and close relatedness or operation of similar selection mechanism amongst these 16 tribes. Though, the $\chi^{2}$-values for interpopulations comparisons (Table 9) show non-significant differences in the allele frequency distribution among these tribes but within population heterogeneity $\left(\mathrm{H}_{\mathrm{S}}\right)$ has been found to be much larger than between populations $\left(\mathrm{D}_{\mathrm{ST}}\right)$. Different factors of population structure such as population size, density, mating pattern, and microevolutionary forces like differential selection, migration etc., considerably contribute to the within population diversity.

Common language frequently signifies a common origin of two populations (Ruhlen, 1987), and as such languages provides a rough classification of populations (Cavalli-Sforza, 1991). $\quad \chi^{2}$-tests among these three linguistic groups of population show largest heterogeneity within the Dravidian speaking group compared to other Austro-Asiatic or Indo-European speaker, of course, the largest significant heterogeneity may partly be attributed to inclusion of larger number of tribes within the Dravidian group than other two groups. 
Acknowledgements This work was supported by the grants from Indian Statistical Institute and from Deutsche Forschungsgemeinschaft (Wa/22/33-1) Germany.

\section{REFERENCES}

Blake NM, Ramesh A, Vijaykumar M, Murty JS, Bhatia KK (1981): Genetic studies on some tribes of Telengana Region, Andhra Pradesh, India. Acta Anthropogenet 5: 41-56

Bienzle U, Ayeni O, Lucas AO, Luzzatto L (1972): Glucose-6-phosphate dehydrogenase and malaria: Greater resistance of females heterozygous for enzyme deficiency and of males with nondeficient variant. Lancet 1 : 107-110

Cavalli-Sforza LL (1991): Genes, peoples and languages. Sci Am November: 104-110

Census of India (1971): Office of the Director of Census of India, New Delhi

Das SR, Mukherjee DP, Sastry DB (1967): Sickle cell trait in Koraput district and other parts of India. Acta Genet Stat Med 17: 62--69

Das SR, Mukherjee BN, Das SK, Malhotra KC (1972): Four types of genetic variants of LDH found in India. Hum Hered 22: 264 (cited from Harris and Hopkinson, 1976)

Fields RA, Parr CW (1963): Human red cell phosphogluconate dehydrogenase. Nature 200: 890 (cited from Harris and Hopkinson, 1976)

Harris H, Hopkinson DA (1976): Hand book of enzyme electrophoresis in human genetics. NorthHolland Publishing, Amsterdam

Hopkinson DA, Mestriner MA, Cortner J, Harris H (1973): Esterase D: a new human polymorphism. Ann Hum Genet 37: 119 (cited from Harris and Hopkinson, 1976)

ICMR (1986): Reports of tribal studies in Jhabua, Ratlan, Ambikapur, Raipur districts of Madhya Pradesh, Ranchi district of Bihar, Anthropology Unit, Institute of Immunohaematology, Bombay

Joshi SR, Mehta MM, Mehta DM, Bhatia HM (1975): Genetic studies of three tribal groups of Dadar-Nagar Haveli in Western India. Paper presented at II Annual Conference of I.S.H.G. Calcutta

Kar S, Seth S, Seth PK (1992): Prevalence of malaria in AO Nagas and its association with G-6-PD and HbE. Hum Biol 64: 187-197

Kate SL, Mukherjee BN, Malhotra KC, Phadke MA, Mutalik GS, Sainani GS (1978): Red cell glucose-6-phosphate dehydrogenase deficiency and haemoglobin variants among ten endogamous groups of Maharashtra \& West Bengal. Hum Genet 44: 229-343

Kher MN, Solanki BR, Parander CM, Junnarkar RV (1967): G-6-PD deficiency at Nagpur and the surrounding area, a survey. Ind Med Gaz $7: 34-39$

Kompf J, Bissbort S, Gussmann S, Ritter H (1975): Polymorphism of red cell glyoxalase-1 (E.C. 4.4.1.5.). A new genetic marker in man. Hum Genet 27 : 141 (cited from Harris and Hopkinson, 1976)

Motulsky AG, Kampbell-Kraut JM (1961): Population genetics of glucose-6-phosphate dehydrogenase deficiency in red cell. Proc Conf Genetic polymorphism and geographic variation in diseases, Grune and Stratton, New York, pp 159-180

Mukherjee BN (1984): Biochemical genetic markers: Its distribution in Indian tribes. Southeast Asian Anthropol 4: 7-14

Mukherjee BN, Das MK (1990): Spatial distribution of two predominant abnormal haemoglobins$\mathrm{HbE}$ and $\mathrm{HbS}$ in Indian subcontinent. J Indian Anthropol Soc 25: 39-59

Mukherjee BN, Malhotra KC, Das SK, Majumder PP, Roy M, Kate SL (1979): Genetic polymorphism among nine endogamous groups of Maharashtra, India. J Hum Evol 8: 555-566

Mukherjee BN, Reddy AP (1983): Wide spread distribution of $\mathbf{L D H}$ variants in various ethnic groups of India. SAP 5 : 1-12 
Papiha SS, Robert DF, Mukherjee DP, Singh SD, Malhotra M (1978): A genetic survey in the Bhil tribe of Madhya Pradesh, Central India. Am J Phys Anthropol 49: 179-185

Papiha SS, Robert DF, Mishra SC (1988): Serogenetic studies among an urban and two tribal populations of Orissa, India. Ann Hum Biol 15: 143-152

Ramesh A, Murty JS, Blake NM (1979): Genetic studies on Kolams of Andhra Pradesh, India. Hum Hered 29: 147-153

Ruhlen M (1987) A guide to the World's languages. Vol. 1. Classification. Stanford University Press, Stanford, California

Saha N, Kirk RL, Shanbhag S, Joshi SR, Bhatia HM (1974): Genetic studies among the Kadars of Kerala. Hum Hered 24: 198-218

Saha N, Kirk RL, Shanbhag S, Joshi SR, Bhatia HM (1976): Population genetic studies in Kerala and Nilgiris (South-west India). Hum Hered 26: 175-184

Singh IP, Bhasin MK, Walter H, Hilling M, Bhasin V, Singh R (1982): Genetic studies of Phagwalas transhumant and settled Gaddis. II. Serum proteins and red cell polymorphisms. Z Morph Anthropol 73: 175-195

Swallow DM, Povey S, Harris H (1973): Activity of "red cell" acid phosphatase locus in other tissues. Ann Hum Genet 37: 31 (cited from Harris and Hopkinson, 1976)

Vyas GN, Bhatia HM, Sukumaran PK, Balakrishnan V, Sanghvi LD (1962): Study of blood groups, abnormal haemoglobins and other genetical characters in some tribes of Gujrat. Am J Phys Anthropol 20: 255-265

Walter H, Danker Hopfe H, Eberhardt D, Tegeler M, Das MK, Das Ketaki, Bhattacharya SK, Sahu PN, Malhotra KC, Mukherjee BN (1992): Investigation on the variability of blood groups polymorphism among sixteen tribal populations from Orissa, Madhya Pradesh and Maharashtra, India. Z Morph Anthropol 79: 69-94

Vol. 38, No. 3, 1993 